\title{
The Vibration Spectra of Hydrazoic Acid, Methyl Azide, and Methyl Isocyanate
}

\author{
The Thermodynamic Functions of Hydrazoic Acid
}

\author{
Eugene H. Eyster, ${ }^{*}$ Department of Physics, University of Michigan, Ann Arbor, Michigan
}

AND

R. H. Gillette, Department of Chemistry, University of Michigan, Ann Arbor, Michigan

(Received February 5, 1940)

\begin{abstract}
The vibration spectra of hydrazoic acid, methyl azide, and methyl isocyanate have been investigated in the spectral region between 2 and $20 \mu$. Correlation of the spectra of these structurally similar molecules has made possible the determination of all the fundamental frequencies of hydrazoic acid and all but the methyl torsion frequency in the methyl compounds. From these fundamental frequencies and the known rotational constants, the usual thermodynamic functions of hydrazoic acid have been calculated to the harmonic oscillator-rigid rotator approximation. Equilibrium constants for some characteristic reactions have also been obtained.
\end{abstract}

$T$ HE structures of hydrazoic acid ${ }^{1}$ and of methyl azide ${ }^{2}$ have now been determined by physical methods, and can be readily interpreted in the light of the modern structural theory. The vibration spectrum of the acid has also been investigated by Davies, ${ }^{3}$ but he was unable to decide with certainty the magnitude of the two lowest frequencies. Since these two vibrations are confined essentially to the azide group, it was thought that a comparison of the spectra of hydrazoic acid and methyl azide would aid in deciding which of the low frequency bands are fundamentals. Since the structural theory predicts for methyl isocyanate a configuration approximating, though not identical with, that of methyl azide, the investigation of its spectrum was also felt appropriate. This compound is of special interest because Herzberg and Verleger, ${ }^{4}$ from studies of the rotational structure of the third $\mathrm{N}-\mathrm{H}$ harmonic of isocyanic acid, conclude that the structures of the covalent azide and isocyanate groups, at least in the free acids, are radically different.

The present paper describes the spectra of the three above-mentioned compounds, and presents a vibrational analysis which yields all the fundamental frequencies of hydrazoic acid, and

\footnotetext{
* National Research Fellow in Chemistry.

${ }^{1}$ Eyster, J. Chem. Phys. 8, 135 (1940).

2 Pauling and Brockway, J. Am. Chem. Soc. 59, 13

3 Davies, Trans. Faraday Soc. 35, 1184 (1939).

${ }^{4}$ Herzberg and Verleger, Physik. Zeits. 37, 444 (1937).
} (1937). all but the methyl torsion oscillations in the methyl compounds. From these results and the rotational constants of hydrazoic acid, ${ }^{1}$ it has been possible to calculate the thermodynamic functions of the acid, and to conclude that the azide and isocyanate groups are structurally similar.

\section{EXPERIMENTAL}

Hydrazoic acid was prepared by the method of Dennis and Isham $^{5}$ from recrystallized sodium azide. The gas was swept from the reaction vessel through a calcium chloride drying tube, and was collected in a liquid-air trap from which a portion of the crude product was redistilled at low temperatures into a glass storage bulb where the substance was stored at reduced pressure until needed.

Methyl azide was prepared by the methylation of sodium azide as described by Dimroth and Wislicenus. ${ }^{6}$ The crude sample was dried and redistilled before use.

Methyl isocyanate was prepared from potassium cyanate by the method of Slotta and Lorenz, ${ }^{7}$ and was similarly dried and fractionally distilled. In no case were the strong water bands observed in samples of these compounds, nor did their spectra suggest the presence of other impurities.

\footnotetext{
${ }^{5}$ Dennis and Isham, J. Am. Chem. Soc. 29, 216 (1907).

6 Dimroth and Wislicenus, Ber. 38, 1573 (1905).

7 Slotta and Lorenz, Ber. 58, 1320 (1925).
} 


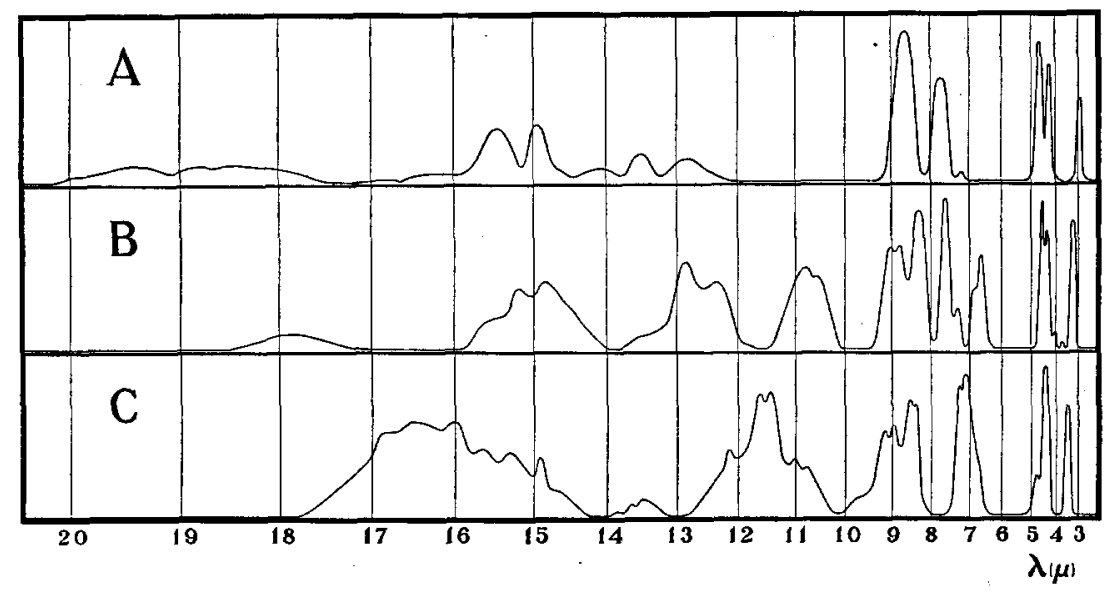

Fig. 1. Percent absorption ( 0 to 100 percent) as a function of wave-length for: $A$, Hydrazoic acid $-13-\mathrm{cm}$ cell $-6 \mathrm{~cm}$ pressure. $B$, Methyl azide $-11-\mathrm{cm}$ cell $-6 \mathrm{~cm}$ pressure. $C$, Methyl isocyanate $-11-\mathrm{cm}$ cell $-8 \mathrm{~cm}$ pressure.

The absorption spectra of these three compounds have been studied between 2 and $20 \mu$ with the potassium bromide prism spectrometer described by Strong and Randall. ${ }^{8}$ This instrument is now completely evacuable, and the thermocouple response, amplified by means of a periodic amplifier, ${ }^{9}$ is automatically recorded. The gases were enclosed in glass absorption cells provided with potassium bromide windows. Hydrazoic acid was studied at pressures of 4 and $6 \mathrm{~cm}$ of $\mathrm{Hg}$ in a 13- $\mathrm{cm}$ cell, methyl azide at a pressure of $6 \mathrm{~cm}$ in an $11-\mathrm{cm}$ cell, and methyl isocyanate at a pressure of $8 \mathrm{~cm}$ in an $11-\mathrm{cm}$ cell. The wave-length calibration has been made by Professor Randall by the measurement of a large number of sharp absorption bands and emission lines whose wave-lengths are known from grating work. The prism spectra so determined are shown in Fig. 1, where the percent absorption is given as a function of wave-length. It has been felt best to present the spectra on the nonlinear wavelength scale which comes from the recorder, in order to show clearly the difficulties attending frequency measurements with an instrument of this type. At wave-lengths less than $4 \mu$ the dispersion has become regrettably small, and beyond $10 \mu$ it is often difficult to choose the center of a band whose envelope is complicated.

As a check on this instrument, three of the hydrazoic acid bands have also been mapped

${ }^{8}$ Strong and Randall, Rev. Sci. Inst. 2, 585 (1931).

${ }^{9}$ Firestone, Rev. Sci. Inst. 3, 163 (1932). with a grating spectrometer described in essentially its present form by Barker and Meyer. ${ }^{10}$ The apparent band centers, as determined by grating and prism instruments, proved to be in satisfactory agreement, especially below $4 \mu$.

The spectra of hydrazoic acid and methyl azide have also been photographed by one of us (E. H. E.) between 7000 and $12,000 \mathrm{~A}$, in the spectroscopic laboratory of the Gates and Crellin Laboratories of Chemistry of the California Institute of Technology. The experimental procedure has already been described in connection with the rotational analysis of the two strong hydrazoic acid bands in this region. ${ }^{1}$ In addition

TABLE I. Infra-red bands and Raman frequencies of hydrazoic acid.

\begin{tabular}{|c|c|c|c|c|}
\hline \multicolumn{3}{|c|}{ INFRA-RED (GAS) } & \multicolumn{2}{|c|}{ RAMAX (LIQUDD) $\dagger$} \\
\hline$\lambda_{\mathrm{VAC}}(\mu)$ & $\operatorname{viC}_{\mathrm{VAC}}\left(\mathrm{CM}^{-1}\right)$ & INTENSITY & $\nu_{\mathrm{VAC}}\left(\mathrm{CM}^{-1}\right)$ & INTENSITY \\
\hline $\begin{array}{l}19.0 \\
15.200 \\
13.536 \\
8.6768^{*} \\
7.880 \\
7.241 \\
4.6720^{*} \\
4.342 \\
2.9980^{*} \\
1.1361 \\
1.047416^{*} \\
0.9353 \\
0.805660^{*}\end{array}$ & \begin{tabular}{|c}
526 \\
657.9 \\
738.8 \\
1152.5 \\
1269.0 \\
1381 \\
2140.4 \\
2303 \\
3335.6 \\
8802 \\
9547.31 \\
10690 \\
12412.19
\end{tabular} & $\begin{array}{l}\text { medium } \\
\text { med. st. } \\
\text { med. st. } \\
\text { very st. } \\
\text { strong } \\
\text { very wk. } \\
\text { strong } \\
\text { med. st. } \\
\text { med. st. } \\
\text { ext. wk. } \\
\text { weak } \\
\text { ext. wk. } \\
\text { very wk. }\end{array}$ & $\begin{array}{l}1300 \\
2389 \text { (?) }\end{array}$ & $\begin{array}{l}\text { weak } \\
\text { very wk. }\end{array}$ \\
\hline
\end{tabular}

* Grating measurements.

$\uparrow$ Engler and Kohlrausch, Zeits. f. physik. Chemie 34B, 214 (1936).

10 Barker and Meyer, Trans. Faraday Soc. 25, 912 (1929). 
to these strong bands, two weaker bands of hydrazoic acid and nine bands of methyl azide were photographed with the prism spectrograph.

The positions of the bands determined by these three methods are listed in Tables I-III. Whenever a band has been measured both with prism and grating instruments, the grating wavelengths are given, but it should be remembered that in only two cases - the 0.8057 and $1.0478 \mu$ bands of hydrazoic acid-have the band centers been located unambiguously by rotational analyses. These tables also give the Raman shifts observed in the liquid phase.

\section{Vibrational Analysis}

It now seems adequately demonstrated that both hydrazoic acid and methyl azide molecules contain linear asymmetric azide groups of essentially the same dimensions, ${ }^{1,2}$ the hydrogen atom or methyl group being situated off the axis of the linear group. Although the interpretations of indirect physical evidence pertaining to the structure of the covalent isocyanate group are at

TABLE II. Infra-red bands and Raman frequencies of methyl azide.

\begin{tabular}{|c|c|c|c|c|}
\hline \multicolumn{3}{|c|}{ INFRA-RED (GAS) } & \multicolumn{2}{|c|}{ RAMAN (LIQUID)* } \\
\hline$\lambda_{\mathrm{VAC}}(\mu)$ & $v_{\mathrm{VAC}}\left(\mathrm{CM}^{-1}\right)$ & INTENSITY & ${ }^{\nu} \mathrm{VAC}\left(\mathrm{CM}^{-1}\right)$ & INTENSITY \\
\hline $\begin{array}{l}8.942 \\
8.375 \\
7.720 \\
7.400 \\
6.972 \\
6.746 \\
4.666 \\
4.484 \\
4.128 \\
3.786 \\
3.3 \\
1.1950 \\
1.1824 \\
1.1620 \\
1.1453 \\
1.1341 \\
0.9480 \\
0.9367 \\
0.9102 \\
0.8938\end{array}$ & $\begin{array}{c}1118 \\
1194 \\
1295 \\
1351 \\
1434 \\
1482 \\
2143 \\
2230 \\
2420 \\
2641 \\
(3000) \\
8369 \\
8457 \\
8606 \\
8731 \\
8818 \\
10,550 \\
10,676 \\
10,987 \\
11,118\end{array}$ & $\begin{array}{l}\text { med. st. } \\
\text { strong } \\
\text { very st. } \\
\text { weak } \\
\text { med. st. } \\
\text { med. st. } \\
\text { very st. } \\
\text { med. st. } \\
\text { very wk. } \\
\text { very wk. } \\
\text { strong }\end{array}$ & $\begin{array}{r}259 \\
668 \\
898 \\
1043 \\
1116 \\
1174 \\
1276 \\
1324 \\
1424 \\
1461 \\
2104\end{array}$ & $\begin{array}{r}164 \\
35 \\
99 \\
5 \\
8 \\
4 \\
42 \\
6 \\
12 \\
16 \\
28\end{array}$ \\
\hline
\end{tabular}

* Kahovec, Kohlrausch, Reitz and Wagner, Zeits. f. phsyik. Chemie 39B, 431 (1938).
TABLE III. Infra-red bands and Raman frequencies of methyl isocyanate.

\begin{tabular}{|c|c|c|c|c|}
\hline \multicolumn{3}{|c|}{ INFRA-RED (GAS) } & \multicolumn{2}{|c|}{ Raman (LIQUid)* } \\
\hline$\lambda_{\mathrm{VAC}}(\mu)$ & $\mathrm{V}_{\mathrm{VAC}}\left(\mathrm{CM}^{-1}\right)$ & INTENSITY & $\nu_{\mathrm{VAC}}\left(\mathrm{CM}^{-1}\right)$ & INTENSITY \\
\hline $\begin{array}{l}16.480 \\
15.356 \\
13.466 \\
11.492 \\
9.030 \\
8.471 \\
7.264 \\
7.084 \\
\text { shelf } \\
4.840 \\
4.480 \\
3.4\end{array}$ & $\begin{array}{r}607 \\
651 \\
743 \\
870 \\
1107 \\
1181 \\
1377 \\
1412 \\
\overline{2066} \\
2232 \\
(2900)\end{array}$ & $\begin{array}{l}\text { strong } \\
\text { med. st. } \\
\text { very wk. } \\
\text { strong } \\
\text { med. st. } \\
\text { strong } \\
\text { med. st. } \\
\text { strong } \\
\text { medium } \\
\text { weak } \\
\text { strong } \\
\text { med. st. }\end{array}$ & $\begin{array}{l}1410 \\
1453\end{array}$ & $\begin{array}{l}5 \\
3 b\end{array}$ \\
\hline
\end{tabular}

* Kopper and Pongratz, Monatshefte 62,78 (1933) ; see also Goubeau, Ber. 68, 912 (1935) for data on the Raman spectrum of methyl iso-

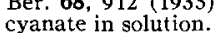

present discordant, ${ }^{4,11}$ the same structural theory which has been so well verified in the azides also predicts a linear structure for the covalent isocyanate group, and places the methyl group off the axis in the methyl derivative. If such be actually the case, the vibration spectra of these three compounds should reflect in their similarity the structural similarity of the molecules, allowance having been made, of course, for the difference between a hydrogen atom and a methyl group. Since these molecules probably all belong at best to the point group $C_{s}$, the usual process of making a vibrational analysis by comparing the Raman and infra-red spectra in the light of the symmetry selection rules is of little value here. Hence it was felt that to establish a definitive vibrational analysis the spectra of hydrazoic acid, methyl azide, and methyl isocyanate should be correlated in that order, and that these, then, should be correlated with the spectrum of a fourth suitable molecule of known structure whose spectrum has been analyzed. For this comparison molecule we have chosen methyl isocyanide. ${ }^{12}$ It has therefore been our aim to discover series of bands in the spectra of these four molecules which might be associated with similar classical vibrations. The series of fundamental bands finally chosen are shown schematically in Fig. 2 and are tabulated in Table IV.

11 Goubeau, Ber. 68, 912 (1935).

${ }^{12}$ Badger and Bauer, J. Am. Chem. Soc. 59, 303 (1937). 


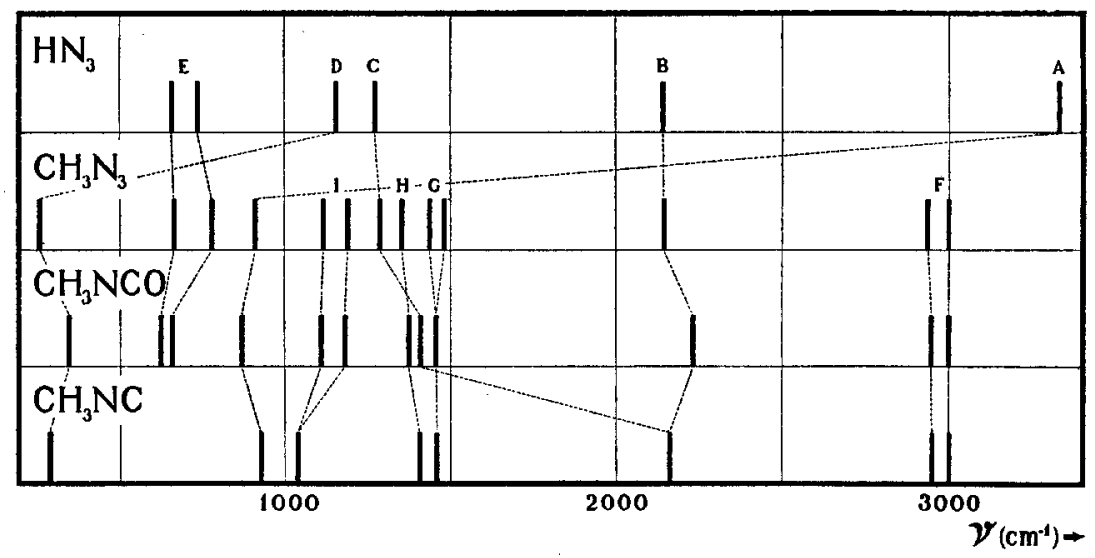

FIG. 2. The series of fundamental frequencies in the spectra of hydrazoic acid, methyl azide, methyl isocyanate, and methyl isocyanide. No attempt has been made to express the intensities.

\section{Fundamental frequencies}

The bands of series $A$ correspond to the stretching of the $\mathrm{N}-\mathrm{H}$ or $\mathrm{N}-\mathrm{CH}_{3}$ bond, and are strong in the infra-red. This series is also strong in the Raman spectra of the methyl compounds, but is not observed in the Raman spectrum of hydrazoic acid, possibly because of association in the liquid which involves the hydrogen atom.

The bands of series $B$ and $C$ correspond to stretching frequencies localized in the linear triatomic groups, degenerating in the isocyanide to a single $\mathrm{N}-\mathrm{C}$ stretching frequency. The asymmetric frequencies of series $B$ are all strong in the infra-red, and can be clearly differentiated from weaker combination and harmonic bands in the vicinity. Though this frequency is of medium intensity in the Raman spectrum of methyl azide, it does not appear in the isocyanate, a fact which we shall later consider. The series $C$ lines are very strong in the Raman spectrum, and thus can be separated without confusion from the series $H$ and $G$ lines which are very much weaker.

Series $D$ corresponds to the bending of the $\mathrm{N}-\mathrm{H}$ or $\mathrm{N}-\mathrm{CH}_{3}$ bond against the linear group. The strong $1152.5 \mathrm{~cm}^{-1}$ band in the infra-red spectrum of hydrazoic acid is identified with this series by the fact that its second harmonic at $2303 \mathrm{~cm}^{-1}$ has been shown to vanish when the hydrogen is substituted with deuterium. ${ }^{13}$ This

${ }^{13}$ Buswell, McMillan, Rodebush and Wall, J. Am. Chem. Soc. 61, 2809 (1939). These workers did not study the fundamental itself, but the evidence is clear from the effect on the harmonic. series is determined by the Raman spectrum alone, in the case of the methyl compounds, as the frequencies are too low for the potassium bromide instrument.

Series $E$ includes the bands arising from the deformation of the linear triatomic group; the twofold degeneracy of such a frequency seems to have been raised by the presence of the off-axis group, and two bands are found in the azides. In hydrazoic acid the band envelopes are well shown, and it appears quite certain that the doublet band with center at $657.9 \mathrm{~cm}^{-1}$ and doublet separation of $22.4 \mathrm{~cm}^{-1}$ is to be interpreted as the parallel component of the $A^{\prime}$ hybrid band. The wider band with the three apparent maxima appears to be the poorly resolved pure perpendicular $A^{\prime \prime}$ band, whose very sharp ${ }^{P} Q$ and ${ }^{R} Q$ branches have been washed out by the wide slits, for the separation of the three maxima is in agreement with the envelopes of the ${ }^{P} P,{ }^{R} P,{ }^{P} R$, and ${ }^{R} R$ branches, as calculated from the known rotational constants of the molecule. The corresponding bands in methyl azide are strong and well separated in the infra-red. In methyl isocyanate these two bands have fused to one very wide band, the centers of whose components can only be estimated.

The bands of series $F$ through $H$ correspond to the well-known internal vibrations of the methyl group, and only series $G$ needs any comment. This series corresponds to the doubly degenerate bending frequency of the free methyl group, and its degeneracy seems to be raised in methyl 
azide. The series $G$ bands in methyl isocyanate appear only as a shelf on the short wave-length side of the strong series $C$ band, but the Raman line at $1453 \mathrm{~cm}^{-1}$ is said to be broad, perhaps indicating a slight raising of the degeneracy here also.

The series $I$ bands, almost identical in appearance in both the azide and isocyanate, correspond to the rocking frequencies of the methyl group. The degeneracy of the frequency in methyl isocyanide is raised in the bent molecules to give two bands at somewhat higher frequencies. A final series, $J$, should occur in the bent methyl compounds, but has not been observed. It corresponds to the torsion oscillation of the methyl group.

\section{Combination, harmonic, and difference bands}

When the fundamental frequencies of a molecule have been correctly assigned, it should be possible to explain all other observed bands as difference transitions or transitions to harmonic and combination states. Actually, with the very high frequencies in the photographic region, it is of ten very difficult to do this uniquely, and even with the lower frequencies unique assignments are not easy to make when there are many fundamentals and low symmetry. Table V summarizes a suggested interpretation of the remainder of the observed spectra of these molecules in terms of the fundamentals of Table IV. Though the methyl torsion frequency is not known for the methyl compounds, it is, unfortunately, rarely involved in strong combination or harmonic bands. Though these assignments do not pretend to be unequivocal, it is interesting to note that no band or Raman line exists which cannot be correlated with the fundamentals of Table IV, with the single exception of the line at $2389 \mathrm{~cm}^{-1}$ in the Raman spectrum of liquid hydrazoic acid. However this line was found on but one plate, and it is stated that the exciting radiation appeared to produce so strong a photochemical reaction that crystals, presumably of some polymer, formed on the walls of the Raman tube toward the end of the exposure. Under such circumstances it is possible that this is not a hydrazoic acid line at all.

\section{Structural Conclusions}

Since it is already known that the structures of methyl azide and hydrazoic acid are similar, the close correspondence of their vibration spectra,

TABLE IV. Series of fundamental frequencies in hydrazoic acid, methyl azide, methyl isocyanate, and methyl isocyanide.

\begin{tabular}{|c|c|c|c|c|c|c|c|c|}
\hline \multirow[b]{2}{*}{ Series } & \multicolumn{2}{|c|}{$\mathrm{HN}_{3}$} & \multicolumn{2}{|c|}{$\mathrm{CH}_{3} \mathrm{~N}_{3}$} & \multicolumn{2}{|c|}{$\mathrm{CH}_{3} \mathrm{NCO}$} & \multicolumn{2}{|c|}{$\mathrm{CH}_{3} \mathrm{NC}^{*}$} \\
\hline & $\nu_{\mathrm{VAC}}\left(\mathrm{CM}^{-1}\right)$ & I.R. & $\nu_{\mathrm{VAC}}\left(\mathrm{CM}^{-1}\right)$ & I.R. & $\nu_{\mathrm{VAC}}\left(\mathrm{CM}^{-1}\right)$ & I.R. & $\nu_{\mathrm{VAC}}\left(\mathrm{CM}^{-1}\right)$ & I.R. \\
\hline$A$ & 3335.6 & $A^{\prime}$ & 914 & $A^{\prime}$ & 870 & $A^{\prime}$ & 928 & $A_{1}$ \\
\hline$B$ & 2140.4 & $A^{\prime}$ & 2143 & $A^{\prime}$ & 2232 & $A^{\prime}$ & \multirow{2}{*}{2161} & \multirow{2}{*}{$A_{1}$} \\
\hline$C$ & 1269.0 & $A^{\prime}$ & 1295 & $A^{\prime}$ & 1412 & $A^{\prime}$ & & \\
\hline$D$ & 1152.5 & $A^{\prime}$ & 259 & $A^{\prime}$ & 353 & $A^{\prime}$ & 290 & $E$ \\
\hline$E$ & $\begin{array}{l}738.8 \\
657.9\end{array}$ & $\begin{array}{l}A^{\prime \prime} \\
A^{\prime}\end{array}$ & $\begin{array}{l}790 \\
654\end{array}$ & $\begin{array}{l}A^{\prime \prime} \\
A^{\prime}\end{array}$ & $\begin{array}{l}652 \\
607\end{array}$ & $\begin{array}{l}A^{\prime \prime} \\
A^{\prime}\end{array}$ & & \\
\hline$F$ & & & $\begin{array}{c}(3000) \\
2931\end{array}$ & $A_{A^{\prime}}^{A^{\prime \prime}}$ & $\begin{array}{l}2994 \\
2951\end{array}$ & $\begin{array}{c}A^{\prime}, A^{\prime \prime} \\
A^{\prime \prime}\end{array}$ & $\begin{array}{l}3003 \\
2951\end{array}$ & $\begin{array}{l}E \\
A_{1}\end{array}$ \\
\hline$G$ & & & $\begin{array}{l}1482 \\
1434\end{array}$ & $\begin{array}{l}A^{\prime} \\
A^{\prime \prime}\end{array}$ & 1453 & $A^{\prime}, A^{\prime \prime}$ & 1456 & $E$ \\
\hline$H$ & & & 1351 & $A^{\prime}$ & 1377 & $A^{\prime}$ & 1414 & $A_{1}$ \\
\hline$I$ & & & $\begin{array}{l}1194 \\
1118\end{array}$ & $\begin{array}{l}A^{\prime \prime} \\
A^{\prime}\end{array}$ & $\begin{array}{l}1181 \\
1107\end{array}$ & $\begin{array}{l}A^{\prime \prime} \\
A^{\prime}\end{array}$ & 1041 & $E$ \\
\hline$J$ & & & 一 & $A^{\prime \prime}$ & 一. & $A^{\prime \prime}$ & & \\
\hline
\end{tabular}

* Data from reference 12 . 
TABLE V. Harmonic, combination, and difference bands of hydrazoic acid, methyl azide, and methyl isocyanate.

\begin{tabular}{|c|c|c|c|}
\hline COMPOUND & $\begin{array}{c}\text { OB- } \\
\text { SERVED } \\
\text { FRE- } \\
\text { QUENCY }\end{array}$ & $\begin{array}{l}\text { OB- } \\
\text { SERVED } \\
\text { IN: }\end{array}$ & SUGgESTED ASSIGNMENT \\
\hline $\mathrm{HN}_{3}$ & $\begin{array}{c}1381 \\
2303 \\
8802 \\
9547.31 \\
10690 \\
12412.19 \\
526\end{array}$ & $\begin{array}{l}\text { Infra-red } \\
\text { Infra-red } \\
\text { Infra-red } \\
\text { Infra-red } \\
\text { Infra-red } \\
\text { Infra-red } \\
\text { Infra-red }\end{array}$ & $\begin{array}{l}(657.9+738.8)=1396.7 \\
(2 \times 11.52 .5)=2305.0 \\
(2 \times 3335.6+2 \times 1152.5)=8827.3^{*} \\
(3 \times 3335.6) \dagger \\
(3 \times 3335.6+1152.5)=10699.8^{*} \\
(4 \times 3335.6) \dagger \\
(1269.0-738.8)=530.2 \\
(1152.5-657.9)=494.6\end{array}$ \\
\hline $\mathrm{CH}_{3} \mathrm{~N}_{3}$ & $\left.\begin{array}{r}550 \\
1043 \\
2230 \\
2420 \\
2641 \\
8369 \\
8457 \\
8606 \\
8731 \\
8818 \\
10550 \\
10676 \\
10987 \\
11118\end{array}\right\}$ & $\begin{array}{l}\text { Infra-red } \\
\text { Raman } \\
\text { Infra-red } \\
\text { Infra-red } \\
\text { Infra-red } \\
\text { Infra-red } \\
\text { Infra-red } \\
\text { Infra-red } \\
\text { Infra-red } \\
\text { Infra-red } \\
\text { Infra-red } \\
\text { Infra-red } \\
\text { Infra-red } \\
\text { Infra-red }\end{array}$ & $\begin{array}{l}(2 \times 259)=518 \\
(1295-259)=1036 \\
2 \times 1118)=2236 \\
(1118+1295)=2413 \\
(1351+1295)=2646 \\
\text { A complex of bands involving } \\
3 \times F \text { fundamentals and also such } \\
\text { series combinations as } 2 \times F+2 \times G \\
\text { etc. More specific assignments are } \\
\text { impossible. } \\
3 \times F+B \\
4 \times F\end{array}$ \\
\hline $\mathrm{CH}_{3} \mathrm{NCO}$ & $\begin{array}{r}743 \\
2066\end{array}$ & $\begin{array}{l}\text { Infra-red } \\
\text { Infra-red }\end{array}$ & $\begin{array}{l}(1107-353)=754 \\
(1377+652)=2029 \\
(1453+606)=2059\end{array}$ \\
\hline
\end{tabular}

* The anharmonicity in the 3335.6 frequency has been taken into account in evaluating the expressions in parentheses.

$t$ These frequencies have already been discussed; see reference 1.

though gratifying, is only useful insofar as it makes possible the choice of low frequency fundamentals. It is, however, of more interest to note the close correspondence between the spectra of methyl azide and methyl isocyanate. From this similarity it seems safe to conclude that the $\mathrm{N}-\mathrm{C}-\mathrm{O}$ group, like the $\mathrm{N}-\mathrm{N}-\mathrm{N}$ group, is linear, and that the methyl group is attached off the axis. The increase in the $B$ and $C$ series frequencies on going from the azide to the isocyanate indicates a strengthening of the binding, and the absence of the series $B$ band in the Raman spectrum of the isocyanate bespeaks a sort of pseudo-symmetry which might be achieved if the $\mathrm{C}-\mathrm{O}$ bond were somewhat stronger than the $\mathrm{N}-\mathrm{C}$ bond. It is probable that the ground state of a molecule $R \mathrm{NCO}$ is attained by resonance among the three electronic structures:<smiles>[R]N=C=[O+]</smiles>

I<smiles></smiles>

II<smiles>[R][N+]#[C-]</smiles>

III
Each of these three structures, if realized alone, would lead to a linear $\mathrm{N}-\mathrm{C}-\mathrm{O}$ group; structure III alone would place the $R$ group on the axis. Although it is difficult to predict how much each of these structures will contribute to the ground state, one might guess, in the absence of any other information, that they contribute equally since each contains the same number of covalent bonds. Upon this assumption, one may easily calculate the distances in the isocyanate group by Pauling's method. ${ }^{14}$ This leads to the result: $\mathrm{N}-\mathrm{C}=1.20 \mathrm{~A} ; \mathrm{C}-\mathrm{O}=1.16 \mathrm{~A}$. Thus one could predict (from Badger's rule, for example) that the $\mathrm{C}-\mathrm{O}$ force constant will be greater than the $\mathrm{N}-\mathrm{C}$ force constant; this difference in the force constants would tend to counteract the mass asymmetry and thus would produce the dynamical pseudo-symmetry mentioned above. Furthermore, the existence of three resonating structures in the isocyanate group as compared to two in the azide group would lead to increased binding energy in the isocyanate and hence to the observed increase in the series $B$ and $C$ frequencies. An electron diffraction study of methyl isocyanate is projected for the near future, and it is hoped that the results will lead to a more complete understanding of the structure of this molecule.

\section{Thermodynamic Functions of Hydrazoic Acid}

The fundamental frequencies of hydrazoic acid, together with the known moments of inertia in the ground state, ${ }^{1}$ provide sufficient data for the calculation of thermodynamic quantities in the rigid rotator-harmonic oscillator approximation. These are perhaps of special interest for this compound since its explosive nature renders calorimetric determinations difficult. The entropy, $S$, the free energy, $F$, and the heat capacity at constant pressure, $C_{n}$, have been calculated by methods adequately described in the literature. The fundamental frequencies are those of Table IV, while the moments of inertia were taken as $I_{A}{ }^{0}=70.75, \quad I_{B}{ }^{0}=69.38$, and $I_{C^{0}}=1.3759$ (in units of $10^{-40} \mathrm{~g} \mathrm{~cm}^{2}$ ). Recent investigations have made it clear that the previously accepted values of the fundamental constants $e$ (the electronic charge) and $h$ (Planck's constant), and hence those constants derived from them, are in error. The magnitudes of the indicated changes are such as to introduce a

${ }^{14}$ Pauling, The Nature of the Chemical Bond (Cornell University Press, 1939), p. 180. 
significant error into the values of thermodynamic functions derived from spectral data. We choose to employ the values: $h=6.6260 \times 10^{-27} \mathrm{erg}$ sec., $e=4.8029 \times 10^{-10}$ abs. e.s.u., and the derived constants $N=6.0228 \times 10^{23}, \quad k=1.3804 \times 10^{-16} \mathrm{erg}$ deg. ${ }^{-1}$, and $h c / k=1.4391 \mathrm{~cm}$ deg. ${ }^{15}$ Detailed calculations show that the rotational and translational entropy is decreased by about 0.07 unit by the introduction of these values, while the rotational and translational contribution to $-\left(F^{0}-H_{0}{ }^{0}\right) / T$ is increased by about 0.06 unit. The change in the vibrational contributions is small in hydrazoic acid because there are no very low frequencies.

The results of the calculations are given in Table VI, where the usual conventions regarding symbols have been observed, except that $E_{0}{ }^{0}$ has been replaced by $H_{0}{ }^{0}$ in accord with more recent notation. ${ }^{16}$ It was thought useless to carry the results above $600^{\circ} \mathrm{K}$, since it is hard to see how $\mathrm{HN}_{3}$ could be important in any equilibria above that temperature.

The main sources of uncertainty in the results of Table VI are (1) neglect of anharmonic terms in the vibrational energy, (2) use of the approximate rotational partition function $Q_{R}=\pi^{\frac{1}{2}}\left(8 \pi^{2} k T\right)^{\frac{3}{2}}\left(I_{A} I_{B} I_{C}\right)^{\frac{1}{2}} / \sigma h^{3}$, and (3) neglect of rotational distortion terms in the rotational energy. The first of these will be negligible in the

15 These are either the base constants chosen by DuMond, or values derived with their aid, and we impute to them no more absolute significance than would he; they have been chosen for convenience, and because they are in essential agreement with "best values" chosen by others. See: DuMond, Phys. Rev. 56, 153 (1939); Wensel, Nat. Bur. Stand. J. Research 22, 375 (1939); and Skiba, Sci. Papers of the I. P. C. R., Tokyo, 34, 1308 (1939).

${ }_{16}$ Giauque and Kemp, J. Chem. Phys, 6, 40 (1938). temperature region concerned, since the anharmonic hydrogen frequencies make very small contributions. The second approximation will become good at temperatures around $300^{\circ} \mathrm{K}$, as shown by Gordon, ${ }^{17}$ but may cause the low temperature results to be inaccurate. The most serious source of uncertainty is probably the third; these rotational distortion terms will cause the rotational levels to be drawn together, and hence our partition functions will be slightly small. It should be noted, however, that it has been necessary in this case neither to guess the moments of inertia nor to resort to the Raman spectrum of the liquid for any of the low frequency vibrations which contribute most to the vibrational partition functions.

Murphy has given analytic expressions for the various thermodynamic functions. ${ }^{18}$ Although these have been extended by Linnett and Avery, ${ }^{19}$ it was thought unnecessary to use the more complicated expression in this case since the vibrational contributions are relatively small. Hence an expression of the simpler form was fitted to the vibrational contributions to $-\left(F^{0}-H_{0}{ }^{0}\right) / T$ of Table VI by the method of averages. This was then combined with the expression for the rotational and translational contributions to give the complete function. In principle, the entropy and other thermodynamic functions could be obtained from this equation by differentiation, but in practice the errors multiply so rapidly that the heat capacities so obtained are practi-

${ }^{17}$ Gordon, J. Chem. Phys. 2, 65 (1934).

18 Murphy, J. Chem. Phys. 5, 637 (1937).

19 Linnett and Avery, J. Chem. Phys. 6, 686 (1938).

TABLE VI. Thermodynamic functions of hydrazoic acid.

\begin{tabular}{l|c|c|c|c|c|c|c|c}
\hline \hline$T\left({ }^{\circ} \mathrm{K}\right)$ & $-\left(\frac{F^{0}-H_{0}^{0}}{T}\right)_{\mathrm{TR}}^{\mathrm{TR}}$ & $-\left(\frac{F^{0}-H_{0}^{0}}{T}\right)_{\mathrm{VIR}}$ & $-\left(\frac{F^{0}-H_{0^{0}}}{T}\right)_{\mathrm{TOTAL}}$ & $S_{\mathrm{TR}}+S_{\mathrm{ROT}}$ & $S_{\mathrm{VIB}}$ & $S_{\mathrm{TOTAL}}$ & $C_{p}$ \\
\hline 100 & 39.402 & 0.000 & 39.402 & 47.348 & 0.000 & 47.348 & 0.014 & 7.960 \\
150 & 42.623 & 0.004 & 42.627 & 50.569 & 0.039 & 50.608 & 0.226 & 8.172 \\
200 & 44.909 & 0.026 & 44.935 & 52.855 & 0.163 & 53.018 & 0.723 & 8.669 \\
250 & 46.682 & 0.070 & 46.752 & 54.628 & 0.401 & 55.029 & 1.384 & 9.330 \\
298.1 & 48.038 & 0.155 & 48.238 & 56.028 & 0.707 & 56.735 & 2.079 & 10.025 \\
300 & 48.131 & 0.158 & 48.289 & 56.077 & 0.720 & 56.797 & 2.100 & 10.046 \\
350 & 49.356 & 0.258 & 49.614 & 57.302 & 1.089 & 58.381 & 2.793 & 10.739 \\
400 & 50.417 & 0.382 & 50.799 & 58.363 & 1.504 & 59.867 & 3.451 & 11.397 \\
450 & 51.353 & 0.532 & 51.885 & 59.298 & 1.943 & 61.241 & 4.061 & 12.007 \\
500 & 52.190 & 0.672 & 52.862 & 60.136 & 2.410 & 62.546 & 4.606 & 12.552 \\
550 & 52.947 & 0.877 & 53.824 & 60.893 & 2.873 & 63.766 & 5.103 & 13.049 \\
600 & 53.639 & 1.062 & 54.701 & 61.584 & 3.331 & 64.915 & 5.558 & 13.504 \\
& & & & & & \\
\hline \hline
\end{tabular}


TABLE VII. The standard free energy and equilibrium constants for the reaction $\frac{1}{2} \mathrm{H}_{2}+\frac{3}{2} \mathrm{~N}_{2}=\mathrm{HN}_{3}$.

\begin{tabular}{l|r|r|r}
\hline$T\left({ }^{\circ} \mathrm{K}\right)$ & $\Delta\left(F^{0}-H_{0^{0}}\right.$ & $\Delta F^{0}$ & $K_{p}$ \\
\hline 298.1 & 6627 & 78525 & $2.655 \times 10^{-58}$ \\
300 & 6680 & 78577 & $5.451 \times 10^{-58}$ \\
400 & 9494 & 81392 & $3.258 \times 10^{-45}$ \\
500 & 12383 & 84281 & $1.403 \times 10^{-37}$ \\
600 & 15280 & 87178 & $1.713 \times 10^{-32}$ \\
\hline
\end{tabular}

cally useless. The final equations are:

$$
\begin{aligned}
-\left(F^{0}-H_{0}{ }^{0}\right) / T= & 250.933 / T \\
& +23.444 \\
& +6.891 \times 10^{-6} T^{2}-9.1682 \\
S^{0}=23.444 & \log T-0.0138 T \\
& +2.097 \times 10^{-7} T^{2}+1.0135
\end{aligned}
$$

The maximum deviations of these expressions from the calculated points are 0.02 and 0.04 , respectively. In order to obtain a reasonably accurate temperature function for $C_{p}$, a quadratic function was fitted to the data of Table VI by the method of least squares; the result is :

$$
C_{p}=5.147+0.01896 T-8.348 \times 10^{-6} T^{2} .
$$

The maximum deviation from the tabular values is $0.04 \mathrm{cal} . / \mathrm{deg}$. above $250^{\circ} \mathrm{K}$.

The thermodynamic functions calculated above may be combined with those of nitrogen ${ }^{20}$ and hydrogen ${ }^{21}$ to give the standard free energy of formation of $\mathrm{HN}_{3}$. It is necessary, however, to evaluate $\Delta H_{0}{ }^{0}$, for which purpose accurate thermal data are very scarce. Meyer ${ }^{22}$ has measured calorimetrically the heat of the reaction:

$$
\mathrm{HN}_{3}=\frac{1}{2} \mathrm{H}_{2}+\frac{3}{2} \mathrm{~N}_{2} \text {. }
$$

He gives $\Delta E=70900 \pm 500 \mathrm{cal}$. at "room temperature." This value, when combined with the heat of solution, is in poor agreement with a value given by Berthelot ${ }^{23}$ for the heat of formation of the aqueous solution. This latter value, however, is based on the heat of combustion of solid ammonium azide and hence may be open to question. If one assumes Meyer's value to apply at $298^{\circ} \mathrm{K}$, one may calculate, from the well-

${ }^{20}$ Giauque and Clayton, J. Am. Chem. Soc. 55, 4875 (1933).

${ }^{21}$ Giauque, J. Am. Chem. Soc. 52, 4816 (1930).

${ }_{22}^{2}$ Gunther, Meyer and Muller-Skold, Zeits. f, physik. Chemie A175, 154 (1935).

${ }^{23}$ Berthelot, Thermochemie (Paris, 1897), Vol. 2, p. 72. known relation:

$$
\Delta H_{0}{ }^{0}=\Delta E^{0}+\Delta n R T-\Delta\left(F^{0}-H_{0}{ }^{0}\right)-T \Delta S^{0},
$$

the value $\Delta H_{0}{ }^{0}=71,898 \mathrm{cal}$. for the formation of $\mathrm{HN}_{3}$ from the gaseous elements. The values of $\Delta F^{0}$ and $K_{p}$ for this reaction given in Table VII have been obtained with the use of this value. Because of the uncertainty in $\Delta H_{0}{ }^{0}$ it was thought meaningless to correct the values of the thermodynamic functions of nitrogen and hydrogen for the change in fundamental constants.

The reaction $\frac{1}{3} \mathrm{NH}_{3}+4 / 3 \mathrm{~N}_{2}=\mathrm{HN}_{3}$ is also of interest. $\Delta F^{0}$ and $K_{p}$ for this reaction may be obtained by combining the above data with those of Stephenson and McMahon ${ }^{24}$ for ammonia. $\Delta H_{0}{ }^{0}$ may be calculated from the following reactions :

$$
\begin{array}{ll}
\frac{1}{2} \mathrm{H}_{2}+\frac{3}{2} \mathrm{~N}_{2}=\mathrm{HN}_{3} & \Delta H_{0}{ }^{0}=71,898 \mathrm{cal} . \\
\frac{1}{3} \mathrm{NH}_{3}=\frac{1}{6} \mathrm{~N}_{2}+\frac{1}{2} \mathrm{H}_{2} & \Delta H_{0}{ }^{0}=3058 \mathrm{cal} . \\
\hline{ }_{3}^{1} \mathrm{NH}_{3}+4 / 3 \mathrm{~N}_{2}=\mathrm{HN}_{3} & \Delta H_{0}{ }^{0}=74,983 \mathrm{cal} .
\end{array}
$$

The values of $\Delta F^{0}$ and $K_{p}$ for this reaction are given in Table VIII.

Since the errors in the partition functions for all substances involved in the reactions just discussed are known to be relatively small, by far the largest uncertainty in $\Delta F^{0}$ and $K_{p}$ is introduced by the uncertainty in the thermal data. For this reason the values of $\Delta\left(F^{0}-H_{0}{ }^{0}\right)$ are included in

TABLE VIII. The standard free energy and equilibrium constants for the reaction $\frac{1}{3} \mathrm{NH}_{3}+4 / 3 \mathrm{~N}_{2}=\mathrm{HN}_{3}$.

\begin{tabular}{l|c|c|c}
\hline$T\left({ }^{\circ} \mathrm{K}\right)$ & $\Delta\left(F^{0}-H_{0}{ }^{0}\right)$ & $\Delta F^{0}$ & $K_{p}$ \\
\hline 298.1 & 4830 & 79813 & $3.018 \times 10^{-59}$ \\
300 & 4868 & 79851 & $6.414 \times 10^{-59}$ \\
400 & 6866 & 81849 & $1.834 \times 10^{-45}$ \\
500 & 8899 & 83882 & $2.097 \times 10^{-37}$ \\
600 & 10909 & 85892 & $5.038 \times 10^{-32}$ \\
\hline
\end{tabular}

Tables VII and VIII so that a more precise value of $\Delta H_{0}{ }^{0}$ could be easily included when it becomes available. It is interesting to note from the tables that at temperatures below about $450^{\circ} \mathrm{K}$, the equilibrium favors decomposition into nitrogen and hydrogen, while above this temperature decomposition into nitrogen and ammonia might be expected to predominate. Meyer ${ }^{22}$ has shown

${ }^{24}$ Stephenson and McMahon, J. Am. Chem. Soc. 61, 437 (1939). 
that in the vicinity of room temperature hydrazoic acid does in fact decompose quantitatively into nitrogen and hydrogen, although it is probable that equilibrium was not completely established.

The authors are very grateful to Professors H. M. Randall and E. F. Barker, of the Physics Department of the University of Michigan, for the use of the excellent spectroscopic equipment which made these results possible. One of us (E. H. E.) wishes to express his appreciation to Professor Richard M. Badger of the California Institute of Technology, in whose laboratory the photographic investigations were made, and is pleased to acknowledge his special indebtedness to the National Research Council.

\title{
Electronic Absorption Spectra in Solution: with Special Reference to the Continuous Absorption of the Halogens
}

\author{
N. S. Bayliss and A. L. G. Rees* \\ Department of Chemistry, University of Western Australia, Crawley, Western Australia
}

(Received February 23, 1940)

The cage theory of liquids has been applied to find the form of potential function that must be added to the $U(r)$ curves of gaseous molecules to give the $U(r)$ curves of dissolved molecules. It is possible to explain the slight displacement of the absorption maximum in halogens dissolved in nonassociated solvents, and also the change in the symmetry of the absorption curve. The large displace-

$\mathrm{T}$ HE chief experimental differences between electronic absorption spectra in the gaseous state and in solution ${ }^{1}$ are as follows: (a) The total absorption intensity is altered, usually increased, in solution. (b) The absorption maximum is displaced to higher frequencies in solution. The displacement is small or zero in normal, nonassociated solvents; but it is considerable, of the order of magnitude $1000-2000 \mathrm{~cm}^{-1}$, in associated solvents. (c) There is a change in the symmetry of the absorption curve about $\nu_{\max }$. (d) Band systems are modified in appearance, displaced in frequency, and often, as in the case of the halogens, disappear altogether in solution.

In this paper we shall be concerned only with those effects which can be discussed in terms of the $U(r)$ curves. Thus (a), which depends on the electronic part of the matrix component of the transition, will not be discussed. Furthermore,

* At present Beit Research Fellow, Imperial College of Science, London.

"For convenience, we shall use the term "solution" to include cases of absorption by pure liquids, where the absorbing molecule will be termed "solute," and the surrounding molecules "solvent." ment of the maximum in associated solvents is due to a slight compression of the solute molecule by the quasicrystalline structure of such solvents. The relaxation time of the solvent cage is such that band absorption should persist in solution when normal and excited states are of the same size; but that it should disappear when $r_{e}^{\prime}>r_{e}{ }^{\prime \prime}$.

we shall deal mainly with the halogen continua; but the results are of more general application. The above effects have often been attributed rather vaguely to "solvation"; but it is evident that any real solvation in the chemical sense would result in more pronounced differences between gas and solution spectra than are generally observed. There are also two points which are worth noting. The displacement of the absorption maximum seems always to be to higher frequencies, and it is large only in associated solvents. Then there is the fact that band systems such as those of the halogens are not observed in solution, although Raman and infra-red data show that molecules possess vibrational quantization in the liquid state. Rotational quantization is, of course, broken down in the liquid; but it has been shown $n^{2}$ that this is of no importance in discussing continuous spectra.

We shall attempt to express the differences between the potential energy curves of gaseous

${ }^{2}$ G. E. Gibson, O. K. Rice and N. S. Bayliss. Phys. Rev. 44, 193 (1933). 